\title{
UNIVERSAL SPACES FOR LOCALLY FINITE-DIMENSIONAL TYCHONOFF SPACES
}

\author{
M. G. CHARALAMBOUS
}

(Communicated by Dennis Burke)

\begin{abstract}
We prove that the class of all locally finite-dimensional Tychonoff spaces and other related classes have universal elements.
\end{abstract}

\section{INTRODUCTION AND DEFINITIONS}

In this paper all spaces are at least Tychonoff, $\tau$ denotes an infinite cardinal, $N$ the set of positive integers, $I$ the unit interval $[0,1], w X$ and $\beta X$ the weight and Stone-Čech compactification of a topological space $X$, respectively, and $\operatorname{dim} X$ the covering dimension of $X$ defined in terms of cozero covers. For standard results and notation in General Topology and Dimension Theory we refer to $[5,6,12]$. A space $X$ is called locally finite dimensional if it has an open cover $\left\{G_{\lambda}: \lambda \in \Lambda\right\}$ with $\operatorname{dim} G_{\lambda}<\infty$ for each $\lambda \in \Lambda[7,13]$. In view of the known fact that $\operatorname{dim} G \leq \operatorname{dim} X$ whenever $G$ is a cozero set of $X$ [see, e.g., 3, Proposition 1], $X$ is locally finite dimensional iff it has a cozero cover $\left\{G_{\lambda}: \lambda \in \Lambda\right\}$ with $\operatorname{dim} G_{\lambda}<\infty$ for each $\lambda$ in $\Lambda$. We write $\operatorname{loc} \operatorname{dim} X \leq n$ if $X$ has an open (or, equivalently, cozero) cover $\left\{G_{\lambda}: \lambda \in \Lambda\right\}$ with $\operatorname{dim} G_{\lambda} \leq n$ for each $\lambda$ in $\Lambda$. If $X$ is a normal space, since $\operatorname{dim} Z \leq \operatorname{dim} X$ if $Z$ is either closed or cozero in $X$, then $\operatorname{loc} \operatorname{dim} X \leq n$ iff $X$ has an open cover $\left\{G_{\lambda}: \lambda \in \Lambda\right\}$ with $\operatorname{dim} \bar{G}_{\lambda} \leq n$ for each $\lambda$ in $\Lambda$, which is the original definition of loc $\operatorname{dim}$ [4]. Following [10], if $f: X \rightarrow Y$ is continuous, by $W(f)$ we denote the smallest cardinal $\alpha$ for which there exists a space $Z$ of weight $\alpha$ and an embedding $g: X \rightarrow Y \times Z$ with $f=\pi \circ g$, where $\pi$ denotes the canonical projection from $Y \times Z$ onto $Y$.

In this paper we establish the existence of universal spaces for several classes of locally finite-dimensional spaces, in particular, for (i) the class of all locally finite-dimensional spaces and (ii) the class of all locally finite-dimensional spaces that can be mapped by some continuous function $f$ with $W(f) \leq \tau$ into a

Received by the editors July 6, 1987 and, in revised form, April 18, 1988.

1980 Mathematics Subject Classification (1985 Revision). Primary 54F45, 54E15.

Key words and phrases. Covering dimension, local dimension, weight, factorization theorems, Čech-complete, $\sigma$-compact, paracompact $p$-spaces. 
metrizable space of weight $\alpha$. This seems to provide an affirmative answer to Pasynkov's Question 12 in [11].

The results on universal spaces follow from certain factorization theorems that are given in $\S 2$ and are corollaries of the factorization theorem for Dim, the covering dimension function for uniform spaces introduced in [2]. $\operatorname{Dim} X$ is defined in terms of the uniformly open sets of a uniform space $X$, i.e., sets of the form $f^{-1}(0,1]$ for some uniformly continuous $f: X \rightarrow I$. Thus, $\operatorname{Dim} X \leq n$ iff every finite uniformly open cover of $X$ has a finite uniformly open refinement of order $\leq n$. The collection of all uniformly open sets of $X$ is a base for the open sets of $X$ that is closed under finite intersections and countable unions. If $X$ is Lindelöf or a metrizable uniform space or has the Stone-Čech uniformity, i.e., that inherited from $\beta X$, then every cozero set of $X$ is uniformly open and hence $\operatorname{Dim} X=\operatorname{dim} X$.

\section{FACTORIZATION THEOREMS}

In the following result, which is proved in [3], $W(X)$ denotes the weight of a uniform space $X$.

Theorem 1. Let $f: X \rightarrow Y$ be a uniformly continuous function between uniform spaces and $\left\{X_{\lambda}: \lambda<\tau\right\}$ a collection of subspaces of $X$ with $W(Y) \leq \tau$. Then there exists a uniformly continuous $g: X \rightarrow Y \times I^{\tau}$ such that $\pi \circ g=f$, where $\pi$ is the projection of $Y \times I^{\tau}$ onto $Y$, and $\operatorname{Dim} g\left(X_{\lambda}\right) \leq \operatorname{Dim} X_{\lambda}$ for each $\lambda<\tau$.

Proposition 1. Let $f: X \rightarrow Y$ be a continuous function and $\left\{X_{\lambda}: \lambda<\tau\right\}$ a collection of Lindelöf subspaces of $X$ with $w Y \leq \tau$. Then there are continuous functions $g: X \rightarrow Z$ and $h: Z \rightarrow Y$, where $Z=Y \times I^{\tau}$, such that $h \circ g=f$ and $\operatorname{dim} g\left(X_{\lambda}\right) \leq \operatorname{dim} X_{\lambda}$ for each $\lambda<\tau$ (cf. [1, Theorem 2].

Proof. Let $X$ be equipped with its Stone-Čech uniformity. $Y$ can be embedded in $I^{\tau}$ and will be considered to be endowed with the subspace uniformity inherited from $I^{\tau}$ so that $W(Y) \leq \tau$. Now $f$ is uniformly continuous and Theorem 1 supplies a uniformly continuous $g: X \rightarrow Z$ such that $\pi \circ g=f$, where $\pi$ is the projection from $Z=Y \times I^{\tau}$ to $Y$, and $\operatorname{Dim} g\left(X_{\lambda}\right) \leq \operatorname{Dim} X_{\lambda}$ for each $\lambda<\tau$. The result follows if we let $h=\pi$ since each $X_{\lambda}$ and $g\left(X_{\lambda}\right)$ is Lindelöf and $\operatorname{dim} Z=\operatorname{Dim} Z$ if $Z$ is Lindelöf.

A similar argument establishes the following result, which generalizes Theorem 3 of [1] and Theorem 1 of [9], where only normal spaces are considered. Recall that $Y$ is said to be $z$-embedded in $X$ if every cozero subset of $Y$ is of the form $Y \cap G$ for some cozero set $G$ of $X$. Closed subspaces of normal spaces and cozero or Lindelöf subspaces of arbitrary spaces are $z$-embedded.

Proposition 2. Let $f: X \rightarrow Y$ be a continuous function into a metric space $Y$ and $\left\{X_{i}: i \in N\right\}$ a countable collection of z-embedded subspaces of $X$. Then there exist a metric space $Z$ and continuous functions $g: X \rightarrow Z$ and $h: Z \rightarrow Y$ such that $h \circ g=f, \operatorname{dim} g\left(X_{i}\right) \leq \operatorname{dim} X_{i}$ for each $i$ in $N$ and $w g(A) \leq w f(A)$ for every subspace $A$ of $X$ with $f(A)$ infinite [3, Proposition 5]. 
We call a space strongly locally finite dimensional if it has a cozero cover $\left\{G_{i}: i \in N\right\}$ with $\operatorname{dim} G_{i} \leq i$. Every metric locally finite-dimensional space is strongly locally finite dimensional [7], but this is not always true in the class of normal spaces.

Example. Let $M$ be a convergent subspace of $\left[0, \omega_{1}\right) \times I^{N}$, where $\omega_{1}$ is the first uncountable ordinal. This means that for each $x$ in $I^{N}$, there is an $\alpha<\omega_{1}$ such that $\left(\alpha, \omega_{1}\right) \times\{x\} \subset M$. Then $M$ is normal with $\operatorname{dim} M=\operatorname{dim} I^{N}=\infty$, and we can choose $M$ to have loc $\operatorname{dim}=0$ [12, Proposition 5.4.5]. Consider a cozero cover $\left\{G_{i}: i \in N\right\}$ of $M$ and fix $x \in I^{N}$. Then there exist $\alpha<\omega_{1}$ and $i \in N$ with $\left[\alpha, \omega_{1}\right) \times\{x\} \subset G_{i}$ and since $I^{N}$ is first countable and $G_{i}$ open, there is an open neighborhood $V$ of $x$ such that $\left(\left[\alpha, \omega_{1}\right) \times V\right) \cap M \subset G_{i}$. Now $\left(\left[a, \omega_{1}\right) \times V\right) \cap M$ is convergent in $\left[0, \omega_{1}\right) \times V$ and hence $\operatorname{dim} G_{i}=\operatorname{dim} V=\infty$.

Proposition 3. Let $f: X \rightarrow Y$ be a continuous function from a strongly locally finite-dimensional space $X$ into a metric space $Y$. Then there exist a locally finite-dimensional metric space $Z$ and continuous $g: X \rightarrow Z$ and $h: Z \rightarrow Y$ such that $f=h \circ g$ and $w Z \leq w Y$.

Proof. Let $\left\{G_{i}: i \in N\right\}$ be a cozero cover of $X$ with $\operatorname{dim} G_{i} \leq i$, and fix a continuous $g_{i}: X \rightarrow I$ with $G_{i}=g_{i}^{-1}(0,1]$. By Proposition 2, there exist a metric space $Z$ and continuous $g: X \rightarrow Z$ and $h: Z \rightarrow Y \times I^{N}$ such that $h \circ g=f \times \prod_{i=1}^{\infty} g_{i}, w g(X) \leq w Y$ and $\operatorname{dim} g\left(G_{i}\right) \leq i$ for each $i \in N$. We may clearly take $Z=g(X)$ and the result follows as it can be verified that $g\left(G_{i}\right)=h^{-1}\left(\pi_{i}^{-1}(0,1]\right)$, where $\pi_{i}$ denotes the projection of $Y \times I^{N}$ onto its $(i+1)$ th factor, so that $\left\{g\left(G_{i}\right): i \in N\right\}$ is a cozero cover of $Z$ with $\operatorname{dim} g\left(G_{i}\right) \leq i$.

\section{UNIVERSAL SPACES}

From Proposition 3 and a straightforward application of a method due to Pasynkov [8] follows a known result for locally finite-dimensional metric spaces. This was first established by different methods by Wenner [13] for separable metric spaces and by Luxemburg [7] for arbitrary metric spaces.

Proposition 4. The class of all metric locally finite-dimensional spaces of weight $\leq \tau$ has a universal element.

Proof. Let $M$ be a universal space for all metric spaces weight $\leq \tau$. Let $\left\{X_{\lambda}: \lambda \in \Lambda\right\}$ be the collection of all locally finite-dimensional subspaces of $M, X$ their topological sum and $f: X \rightarrow M$ the unique function from $X$ to $M$ whose restriction to each $X_{\lambda}$ is the inclusion of $X_{\lambda}$ into $M$. Then $f$ is continuous, $X$ is locally finite-dimensional and, by Proposition 3, there exist a locally finite-dimensional metric space $Z$ and continuous $g: X \rightarrow Z$ 
and $h: Z \rightarrow M$ with $f=h \circ g$. Now the restriction of $g$ to each $X_{\lambda}$ is an embedding and $Z$ is the required universal space.

The following result will prove useful. Recall that for an open set $G$ of a space $X, E x G$ denotes the biggest open set of $\beta X$ whose intersection with $X$ is $G . \quad E x\left(G_{1} \cap G_{2}\right)=E x G_{1} \cap E x G_{2}$ and, if $G_{1}$ and $G_{2}$ are cozero, $\operatorname{Ex}\left(G_{1} \cup G_{2}\right)=\operatorname{Ex} G_{1} \cup \operatorname{Ex} G_{2}$ [5, Lemma 7.1.13].

Lemma 1. For every cozero set $G$ of a space $X$ and every normal space $H$ with $G \subset H \subset E x G, \operatorname{dim} G=\operatorname{dim} H$.

Proof. Firstly, $\operatorname{dim} G \leq \operatorname{dim} H$ because $G$ is $z$-embedded in $H$ [e.g., 3, Proposition 1]. Conversely, suppose $\operatorname{dim} G=n$ and let $\left\{G_{1}, G_{2}, \ldots, G_{k}\right\}$ be a cozero cover of $H$. Let $\left\{F_{1}, F_{2}, \ldots, F_{k}\right\}$ and $\left\{H_{1}, H_{2}, \ldots, H_{k}\right\}$ be, respectively, a zero and a cozero cover of $H$ with $H_{i} \subset F_{i} \subset G_{i}$. Since $\operatorname{dim} G \leq n$, the cozero cover $\left\{G \cap H_{1}, G \cap H_{2}, \ldots, G \cap H_{k}\right\}$ of $G$ has a cozero shrinking $\left\{V_{1}, V_{2}, \ldots, V_{k}\right\}$ of order $\leq n$. Then $\left\{H \cap \operatorname{ExV}_{1}, H \cap E x V_{2}, \ldots, H \cap E x V_{k}\right\}$ is an open cover of the normal space $H$ and, therefore, it has a cozero shrinking $\left\{W_{1}, W_{2}, \ldots, W_{k}\right\}$. It is readily verified that $H \cap E x V_{i} \subset F_{i}$ and hence $\left\{W_{1}, W_{2}, \ldots, W_{k}\right\}$ is a cozero shrinking of the cozero cover $\left\{G_{1}, G_{2}, \ldots, G_{k}\right\}$ of $H$ of order $\leq n$. Thus, $\operatorname{dim} H \leq n=\operatorname{dim} G$, and the proof is complete.

Proposition 5. The class of all locally finite-dimensional spaces of weight $\leq \tau$ has a universal element that is locally compact.

Proof. Let $\left\{X_{\lambda}: \lambda \in \Lambda\right\}$ be the collection of all locally finite-dimensional subspaces of $I^{\tau}, X_{0}$ their topological sum and $i: \beta X_{0} \rightarrow I^{\tau}$ the unique continuous function whose restriction to each $X_{\lambda}$ is the inclusion of $X_{\lambda}$ into $I^{\tau}$. Since clearly $w X_{\lambda} \leq \tau, X_{\lambda}$ has a cozero cover $\left\{G_{\lambda \alpha}: \alpha<\tau\right\}$ with $\operatorname{dim} G_{\lambda \alpha} \leq n(\alpha)<\infty$. Now for each $\alpha<\tau, G_{\alpha}=\bigcup_{\lambda \in \Lambda} G_{\lambda \alpha}$ is a cozero set of $X_{0}$ with $\operatorname{dim} G_{\alpha} \leq n(\alpha)<\infty$. Let $g_{\alpha}: \beta X_{0} \rightarrow I$ be a continuous function such that $G_{\alpha}=X_{0} \cap H_{\alpha}$, where $H_{\alpha}=g_{\alpha}^{-1}(0,1]$. Then $X_{0}$ is contained in $X=\bigcup\left(H_{\alpha}: \alpha<\tau\right)$ and, by Lemma 1 , since $H_{\alpha}$ is Lindelöf, $\operatorname{dim} H_{\alpha}=\operatorname{dim} G_{\alpha} \leq n(\alpha)<\infty$. Now let $f: X \rightarrow I^{\tau} \times I^{\tau}$ be the restriction to $X$ of $i \times \prod_{\alpha<\tau} g_{\alpha}$ and apply Proposition 1 to obtain a space $Z$ of weight $\leq \tau$ and continuous functions $g: X \rightarrow Z$ and $h: Z \rightarrow I^{\tau} \times I^{\tau}$ with $f=h \circ g$ and $\operatorname{dim} g\left(H_{\alpha}\right) \leq n(\alpha)<\infty$ for $\alpha<\tau$. Note that for $\alpha<\tau$ there is a projection $\pi: I^{\tau} \times I^{\tau} \rightarrow I$ such that $g_{\alpha}=\pi \circ f$ and hence $g\left(H_{\alpha}\right)=h^{-1}\left(\pi^{-1}(0,1]\right) \cap g(X)$ is a cozero set of $g(X)$, which, therefore, belongs to the class of locally finitedimensional spaces of weight $\leq \tau$. Finally, $g(X)$ is a universal element of this class because the restriction of $g$ to each $X_{\lambda}$ is an embedding and $I^{\tau}$ is a universal space for all spaces of weight $\leq \tau$. Furthermore, if $H_{\alpha n}=g_{\alpha}^{-1}(1 / n, 1]$ and $F_{\alpha n}=g_{\alpha}^{-1}[1 / n, 1], n \in N, \alpha<\tau$, it is not difficult to see that $g\left(F_{\alpha n}\right)$ is compact and $\left\{g\left(H_{\alpha n}\right): n \in N, \alpha<\tau\right\}$ is a cozero cover of $g(X)$ with $\frac{\alpha n}{g\left(H_{\alpha n}\right)}$ compact. Hence $g(X)$ is locally compact. 
Only minor adjustments of the same proof suffice to establish the following two results. In the first case, $\left\{H_{\alpha}: \alpha<\tau\right\}$ can be taken to be countable and, in the second case, $n(\alpha)$ a fixed integer $n$.

Proposition 6. The class of all strongly locally finite-dimensional spaces of weight $\leq \tau$ has a universal element that is $\sigma$-compact and locally compact.

Proposition 7. The class of all spaces with $\operatorname{loc} \operatorname{dim} \leq n$ and weight $\leq \tau$ has $a$ universal element that is locally compact.

We can, in fact, improve the last result as follows.

Proposition 8. The class of all spaces with $\operatorname{loc} \operatorname{dim} \leq n$ and weight $\leq \tau$ has a universal element $Z$ which is compact with $\operatorname{dim} Z \leq n$.

Proof. Let $Y$ be the universal element provided by Proposition 7 and let $Z=$ $Y \cup\{\infty\}$ be the one-point compactification of $Y$. If $F$ is a closed set of $Z$ disjoint from $\infty$, then $F$ is a compact subspace of $Y$ so that $\operatorname{dim} F \leq$ $\operatorname{loc} \operatorname{dim} Y \leq n$. This and the normality of $Z$ imply $\operatorname{dim} Z \leq n$ [4], and the result follows.

The same conclusion can be reached through the following result, which is of some interest, and the fact that the class of spaces of weight $\leq \tau$ and $\operatorname{dim} \leq n$ has a compact universal element [8].

Proposition 9. An infinite space $X$ with $\operatorname{loc} \operatorname{dim} X \leq n$ can be embedded in $a$ space $Y=X \cup\{\infty\}$, where $\infty \notin X$, with $\operatorname{dim} Y \leq n$ and $w Y=w X$.

Proof. Let $\left\{G_{\alpha}: \alpha<\tau\right\}$ be a cozero cover of $X$ with $\operatorname{dim} G_{\alpha} \leq n$ for $\alpha<\tau=$ $w X$. Further, let $F_{\alpha i}$ be zero sets and $G_{\alpha i}$ be cozero sets of $X, i \in N$, with $F_{\alpha i} \subset G_{\alpha i} \subset F_{\alpha i+1}$ and $G_{\alpha}=\bigcup_{i=1}^{\infty} F_{\alpha i}=\bigcup_{i=1}^{\infty} G_{\alpha i}$. We define a subset $G$ of $Y$ to be open if either $G$ is an open subset of $X$ or $Y-G$ is a closed subset of $\bigcup\left(F_{\alpha k}: \alpha \in J\right)$ for some $k \in N$ and a finite subset $J$ of $\tau$. Clearly, $w Y=\tau$. Consider a continuous $f: X \rightarrow I$ with $G_{\alpha i}=f^{-1}(0,1]$ and $F_{\alpha i}=f^{-1}\{1\}$. This can be continuously extended to $Y$ by setting $f(\infty)=0$, for, in that case, the restrictions of $f$ to both of the closed sets $F_{\alpha i+1}$ and $Y-G_{\alpha i}$ are continuous. Hence each $F_{\alpha i}$ is zero in $Y$ and each $G_{\alpha i}$ and $G_{\alpha}$ cozero. Thus $Y$ has a base consisting of cozero sets and is therefore Tychonoff.

Now consider a closed set $F$ of $\beta Y$ disjoint from $\infty$. Let $V$ be an open neighborhood of $F$ in $\beta Y$ with $\infty \notin \bar{V}$ and pick $\alpha_{1}, \ldots, \alpha_{k}<\tau$ with $\bar{V} \cap Y \subset \bigcup_{i=1}^{k} G_{\alpha_{i}}$. Then $F$ is contained in $\bigcup_{i=1}^{k} E x G_{\alpha_{i}}$ and there exists a closed cover $\left\{F_{i}: i=1, \ldots, k\right\}$ of $F$ shrinking $\left\{F \cap E x G_{\alpha_{i}}: i=1, \ldots, k\right\}$. If $H_{i}$ is a cozero set of $\beta Y$ with $F_{i} \subset H_{i}$ and $H_{i} \cap Y \subset G_{\alpha_{i}}$, by Lemma $1 \operatorname{dim} H_{i} \leq n$ and hence $\operatorname{dim} F_{i} \leq n$. Now, by the countable sum theorem, $\operatorname{dim} F \leq n$. Hence $\operatorname{dim} Y=\operatorname{dim} \beta Y \leq n$, and this completes the proof.

For the rest of our results we need a lemma and a simplified version of [3, Theorem 9]. Recall that $b w X$ is the smallest cardinal $\tau$ for which there exists a continuous $f: X \rightarrow M$ with $M$ metrizable and $W(f)=\tau$ [10]. 
Lemma 2. Let $X$ be a locally finite-dimensional space with $b w X \leq \tau$. Then $X$ has a cover of cardinality $\leq \tau$ consisting of cozero sets with $\operatorname{dim}<\infty$.

Proof. We can consider $X$ as a subspace of $M \times I^{\tau}$ for some metrizable $M$. Let $\left\{G_{\lambda i}: \lambda \in \Lambda, i \in N\right\}$ be a $\sigma$-discrete base of $M$ and $\left\{U_{\alpha}: \alpha<\tau\right\}$ a base of $I^{\tau}$ consisting of cozero sets. For each $i$ and $n$ in $N$, let $V_{\alpha, i, n}$ be the union of all sets $\left(G_{\lambda i} \times U_{\alpha}\right) \cap X$ that are contained in some cozero set of $X$ with $\operatorname{dim} \leq n$. Each $V_{\alpha, i, n}$ is the disjoint union of open sets with $\operatorname{dim} \leq n$. Hence $\operatorname{dim} V_{\alpha, i, n} \leq n$ and evidently $\left\{V_{\alpha, i, n}: \alpha<\tau, i, n \in N\right\}$ is the required cover of $X$.

Theorem 2. Let $f: X \rightarrow Y$ be a perfect and uniformly continuous function between uniform spaces such that $Y$ is paracompact and every cozero set of $Y$ is uniformly open. Then $\operatorname{dim} X \leq \operatorname{Dim} X$ [3, Theorem 9].

Proposition 10. The class $\mathscr{C}$ of all locally finite-dimensional spaces that can be mapped by some continuous function $f$ with $W(f) \leq \tau$ into a metrizable space of weight $\theta$ has a universal element which is locally Cech-complete and paracompact.

Proof. Let $M$ be a universal element of the class of all metric spaces of weight $\leq \theta$ which is complete. Every member of $\mathscr{C}$ is homeomorphic with a locally finite-dimensional subspace of $M \times I^{\tau}$. Let $\left\{X_{\lambda}: \lambda \in \Lambda\right\}$ be the collection of all such subspaces, $X_{0}$ their topological sum and $i: \beta X_{0} \rightarrow \beta\left(M \times I^{\tau}\right)$ the unique continuous function whose restriction to each $X_{\lambda}$ is the inclusion of $X_{\lambda}$ into $M \times I^{\tau}$. By Lemma 2, each $X_{\lambda}$ has a cozero cover $\left\{G_{\lambda \alpha}: \alpha<\tau\right\}$ with $\operatorname{dim} G_{\lambda \alpha} \leq n(\alpha)<\infty$. Now each $G_{\alpha}=\bigcup_{\lambda \in \Lambda} G_{\lambda \alpha}$ is a cozero set of $X_{0}$ with $\operatorname{dim} G_{\alpha} \leq n(\alpha)<\infty$ and there exists a continuous $g_{\alpha}: \beta X_{0} \rightarrow I$ such that $G_{\alpha}=g_{\alpha}^{-1}(0,1] \cap X_{0}$. Let $Y=\left(M \times I^{\tau}\right) \times I^{\tau}, f=i \times \prod_{\alpha<\tau} g_{\alpha}: \beta X_{0} \rightarrow$ $\beta\left(M \times I^{\tau}\right) \times I^{\tau}, X=f^{-1}(Y)$ and $H_{\alpha}=g_{\alpha}^{-1}(0,1] \cap X, \alpha<\tau$. Note that $f: X \rightarrow Y$ is perfect, $X$ is a paracompact $p$-space, each $H_{\alpha}$ is paracompact as a cozero set of $X$ and, by Lemma $1, \operatorname{dim} H_{\alpha}=\operatorname{dim} G_{\alpha} \leq n(\alpha)<\infty$.

We now endow $X$ with its finest uniformity, $M$ with that induced by a compatible metric, $I^{\tau}$ with its unique uniformity and $M \times I^{\tau}$ and $Y$ with the resulting product uniformities. Theorem 1 applied to $f: X \rightarrow Y$ yields a uniformly continuous $g: X \rightarrow Y \times I^{\tau}$ with $f=\pi \circ g$, where $\pi$ denotes the projection of $Y \times I^{\tau}$ onto $Y$, and $\operatorname{Dim} g\left(F_{\alpha n}\right) \leq \operatorname{Dim} F_{\alpha n}$, where $F_{\alpha n}=$ $g_{\alpha}^{-1}\left[\frac{1}{n}, 1\right] \cap X$ for each $\alpha<\tau$ and $n$ in $N$. Note that $F_{\alpha n}$ is a $z$-embedded subspace of $X$ and therefore $\operatorname{Dim} g\left(F_{\alpha n}\right) \leq \operatorname{Dim} F_{\alpha n}=\operatorname{dim} F_{\alpha n} \leq \operatorname{dim} H_{\alpha} \leq$ $n(\alpha)$. Now, if $\pi_{\alpha}: Y \rightarrow I$ denotes the projection onto the $\alpha$ th factor of the second copy of $I^{\tau}$ in $Y$, then $g\left(H_{\alpha}\right)=\pi^{-1}\left(\pi_{\alpha}^{-1}(0,1]\right) \cap g(X)$. It follows that, since $f: X \rightarrow Y$ is perfect, the same is true of $g: X \rightarrow g(X), \pi: g(X) \rightarrow Y$ and $\pi: g\left(H_{\alpha}\right) \rightarrow \pi_{\alpha}^{-1}(0,1]$. Hence $g\left(H_{\alpha}\right)$ is Čech-complete and paracompact. Finally, if $\sigma: Y \rightarrow M$ denotes natural projection, it is readily seen that the composite of $\pi: g\left(F_{\alpha n}\right) \rightarrow Y$ with $\sigma$ is perfect and uniformly continuous and, by Theorem 2, $\operatorname{dim} g\left(F_{\alpha n}\right) \leq \operatorname{Dim} g\left(F_{\alpha n}\right) \leq n(\alpha)$ for each $n$ in $N$ and 
$\alpha<\tau$. By the countable sum theorem for $\operatorname{dim}, \operatorname{dim} g\left(H_{\alpha}\right) \leq n(\alpha)$, and it is now clear that $Z=\bigcup_{\alpha<\tau} g\left(H_{\alpha}\right)$ is a universal element of $\mathscr{C}$ which is locally Čech-complete and paracompact.

As with Proposition 5, two more results have almost the same proof. We first point out that a slightly stronger condition is satisfied by $Z$. Let $\alpha_{1}$, $\alpha_{2}, \ldots<\tau, h=\sigma \times \prod_{i=1}^{\infty} \pi_{\alpha_{i}}: Y \rightarrow M \times I^{N}$ and $\sigma_{i}: M \times I^{N} \rightarrow I$ the projection onto the $i$ th factor of $I^{N}$. Then $h$ is perfect and so is its composite with $\pi: g(X) \rightarrow Y$. It follows that

$$
\bigcup_{i=1}^{\infty} g\left(H_{\alpha_{i}}\right)=\pi^{-1}\left(h^{-1}\left(\bigcup_{i=1}^{\infty} \sigma_{i}^{-1}(0,1]\right)\right)
$$

is a Čech-complete paracompact $p$-space. This is exactly what is needed in Proposition 12.

Proposition 11. The class of all spaces with loc dim $\leq n$ that can be mapped by some continuous function $f$ with $W(f) \leq \tau$ into a metrizable space of weight $\theta$ has a universal element which is locally Cech-complete and paracompact.

Proposition 12. The class of all strongly locally finite-dimensional spaces that can be mapped by some continuous function $f$ with $W(f) \leq \tau$ into a metrizable space of weight $\theta$ has a universal element which is a Cech-complete paracompact p-space.

The last result can be rephrased as follows. Recall that $\mu w X$ is the supremum of all cardinals $\alpha$ for which there exists a map from $X$ onto a metrizable space of weight $\alpha[10]$.

Proposition 13. The class of all strongly locally finite-dimensional spaces with $b w \leq \tau$ and $\mu w \leq \theta$ has a universal element which is a Čech-complete paracompact p-space.

Proof. It suffices to prove that for the universal space $Z$ of Proposition 12, $\mu w Z \leq \theta$. This is true because $Z$ can be mapped by a perfect map onto a metric space of weight $\leq \theta$ [10, Proposition 2].

Consider a locally finite-dimensional normal and countably paracompact space $X$ such that $\operatorname{dim} F=\operatorname{loc} \operatorname{dim} F$ for every closed subset $F$ of $X$. Let $\left\{H_{i}: i \in N\right\}$ be an open cover of $X$ with $\operatorname{loc} \operatorname{dim} H_{i} \leq i$. This has a closed shrinking $\left\{F_{i}: i \in N\right\}$, and if $G_{i}$ is a cozero set of $X$ with $F_{i} \subset G_{i} \subset H_{i}$ then $G_{i}$ is the countable union of closed sets of $X$ with $\operatorname{dim}=\operatorname{loc} \operatorname{dim} \leq i$. By the countable sum theorem, $\operatorname{dim} G_{i} \leq i$, and hence $X$ is strongly locally finite dimensional. Thus, Propositions 6 and 13 generalize the results of Bobkov quoted in [11]. 


\section{REFERENCES}

1. A. Arhangel' skii, Factorization of mappings according to weight and dimension, Soviet Math. Dokl. 8 (1967), 731-734.

2. M. G. Charalambous, $A$ new covering dimension function for uniform spaces, J. London Math. Soc. (2) 11 (1975), 137-143.

3. __ Further theory and applications of covering dimension of uniform spaces (to appear).

4. C. H. Dowker, Local dimension of normal spaces, Quart. J. Math. Ser. (2) 6 (1955), 101-120.

5. R. Engelking, General topology, Polish Scientific Publishers, Warsaw, 1977.

6. __ Dimension theory, Polish Scientific Publishers, Warsaw, 1978.

7. L. Luxemberg, On universal metric locally finite-dimensional spaces, General Topology Appl. 10 (1979), 283-290.

8. B. A. Pasynkov, On universal bicompacta of a given weight and dimension, Soviet Math. Dokl. 5 (1964), 245-246.

9. __ A factorization theorem for non-closed sets, Soviet Math. Dokl. 13 (1972), 292-295.

10. __ Factorization theorems in dimension theory, Russian Math. Surveys 36 (1981), 175-209.

11. _ On dimension theory, Aspects of Topology, Cambridge Univ. Press, Cambridge, 1985, pp. 227-250.

12. A. R. Pears, Dimension theory of general spaces, Cambridge Univ. Press, Cambridge, 1976.

13. B. R. Wenner, A universal metric locally finite-dimensional space, Fund. Math. 80 (3) (1973), 283-286.

Department of Mathematics, University of Nairobi, P. O. Box 30197, Nairobi, Kenya 